\title{
Usefulness of the Addition of Renal Function to the CHA2DS2- VASc Score as a Predictor of Thromboembolism and Mortality in Patients Without Atrial Fibrillation
}

\author{
Christine Parsons, MD $^{\mathrm{a}}$, Stephen Cha, $\mathrm{MS}^{\mathrm{b}}$, Win-Kuang Shen, $\mathrm{MD}^{\mathrm{c}}$, Alanna M. Chamberlain, \\ $\mathbf{P h D}^{d}$, Sushil Allen Luis, MBBS ${ }^{e}$, Mira Keddis, $\mathbf{M D}^{\dagger}$, and Fadi Shamoun, MDc \\ aDepartment of Internal Medicine, Mayo Clinic, 13400 E Shea Blvd, Scottsdale, AZ, USA 85259

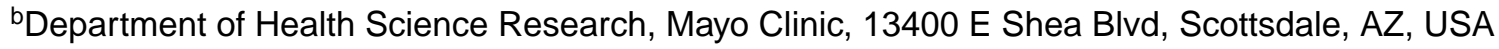 \\ 85259 \\ 'Division of Cardiovascular Diseases, Mayo Clinic, 13400 E Shea Blvd, Scottsdale, AZ, USA \\ 85259 \\ dDepartment of Health Sciences Research, Mayo Clinic, $2001^{\text {st }}$ St SW, Rochester, MN, USA \\ 55905 \\ eDepartment of Cardiovascular Medicine, Mayo Clinic, $2001^{\text {st }}$ St SW, Rochester, MN, USA 55905 \\ fDepartment of Nephrology, Mayo Clinic, 5777 E MayoBlvd, Phoenix, AZ, USA 85054
}

\begin{abstract}
Research is conflicting whether kidney function should be incorporated in thromboembolism risk prediction. Our published data showed that the CHA2DS2-VASc score predicts thromboembolism and mortality in those without atrial fibrillation. We used the Rochester Epidemiology Project medical records system to retrospectively evaluate whether adding renal impairment (1 point) to the CHA2DS2-VASc score (-R) enhances the score's prediction of mortality, thromboembolism, and atrial fibrillation in patients without atrial fibrillation. We identified patients that had an implantable cardiac monitoring device placed between January 1, 2004 and December 31, 2013, which was defined as the start date. Follow-up was through March 7, 2016. An implantable device was required to discern the absence of atrial fibrillation. Renal impairment was defined as chronic kidney disease stage 3 or greater. The population $(n=1,606)$ had a mean age of 69.8 years and median follow-up of 4.8 years. Baseline renal impairment was predictive of mortality (HR 2.06,
\end{abstract}

Corresponding author: Fadi Shamoun, MD, Mayo Clinic, 13400 E Shea Blvd, Scottsdale, AZ, USA 85259,

Shamoun.Fadi@mayo.edu, Phone: (480) 301-6201, Fax: (480) 301-8018.

CONFLICT OF INTEREST

Alanna M. Chamberlain is a Co-Investigator of the Rochester Epidemiology Project. The authors have no other conflicts of interest to declare.

AUTHORSHIP

All authors listed have contributed sufficiently to the project to be included as authors and are responsible for the study design, manuscript content and editorial decisions. All authors reviewed and approved the final version.

Publisher's Disclaimer: This is a PDF file of an unedited manuscript that has been accepted for publication. As a service to our customers we are providing this early version of the manuscript. The manuscript will undergo copyediting, typesetting, and review of the resulting proof before it is published in its final citable form. Please note that during the production process errors may be discovered which could affect the content, and all legal disclaimers that apply to the journal pertain. 
$95 \%$ CI 1.64 to $2.60, P<0.001$ ), thromboembolism (HR $1.34,95 \%$ CI 0.96 to $1.87, P=0.09$ ), and atrial fibrillation (HR 1.31, 95\% CI 0.98 to $1.74, P=0.07$ ). Lower glomerular filtration rate correlated significantly with mortality. Increasing CHA2DS2-VASc-R score correlated significantly with mortality, thromboembolism, and incident atrial fibrillation. The addition of renal impairment to the CHA2DS2-VASc score improved the C-statistics for thromboembolism and survival from 0.72 to $0.73(P=0.01)$ and 0.70 to $0.72(P<0.001)$. Adding renal impairment to the CHA2DS2-VASc score improves the score's prediction of thromboembolism and mortality in a population without atrial fibrillation, although the incremental benefit appears mild.

\section{Keywords}

renal impairment; chronic kidney disease; risk factors; thromboembolism

\section{INTRODUCTION}

The CHA2DS2-VASc score is a well-validated tool to assess the risk of stroke and systemic emboli in patients with non-valvular atrial fibrillation. ${ }^{1,2}$ Atrial fibrillation confers a 6-fold increased risk of stroke, ${ }^{3}$ however, the causality of this relationship may not be as concrete as previously thought. Our recent study shows that the CHA2DS2-VASc score predicts stroke and mortality in a population without atrial fibrillation. ${ }^{4}$ Research is conflicting whether kidney function should be incorporated in thromboembolism risk prediction tools. ${ }^{5-7}$ The addition of renal impairment to the CHADS2 score ( 2 points for glomerular filtration rate $[\mathrm{GFR}]<60 \mathrm{~mL} / \mathrm{min}$ ) had similar predictive value to the CHADS2 and CHA2DS2-VASc scores, although notably this population excluded patients with a GFR $<30 .{ }^{7}$ The ATRIA stroke score, which includes a point for proteinuria and a point for GFR $<45 \mathrm{~mL} / \mathrm{min}$, demonstrated superior stroke prediction compared to the CHA2DS2-VASc score although $\mathrm{C}$-indices were similar. ${ }^{8}$ The present study aims to evaluate whether adding renal impairment (1 point) to the CHA2DS2-VASc score (-R) predicts thromboembolic events or mortality in a population without atrial fibrillation with implantable cardiac monitoring devices.

\section{METHODS}

We conducted a retrospective review utilizing the Rochester Epidemiology Project, which links and indexes the medical records of residents in Olmsted County, Minnesota. ${ }^{9}$ The Olmsted Medical Center and Mayo Clinic Institutional Review Boards approved the study. We identified all subjects with an implantable cardiac monitoring device (pacemaker, defibrillator or loop recorder) placed between January 1, 2004 and December 31, 2013. The index date (time 0 ) was defined as the date of device implantation and follow-up was defined as the time to first thromboembolic event, death or the end of the study period (March 7, 2016). Implantable cardiac devices allow continuous and accurate monitoring of dysrhythmias, ${ }^{10}$ and were required to better exclude those with atrial fibrillation.

Inclusion criteria included age $>50$ years and no prior diagnosis of atrial fibrillation. A total of 1,606 subjects met inclusion criteria: 3,033 patients were identified as having a qualifying implantable device and 1,427 patients were subsequently removed as they had an 
International Classification of Diseases, Ninth Revision, (ICD-9) diagnosis of atrial fibrillation prior to device implantation. Figure 1 describes the process for generating the cohort. ICD-9 diagnostic codes were used to identify the baseline individual CHA2DS2VASc components and renal impairment. Renal impairment was defined as CKD stage 23 , including end stage renal disease or dialysis. Baseline creatinine was also obtained from inpatient or outpatient laboratory records and used to calculate the GFR $\left(\mathrm{mL} / \mathrm{min} / 1.73 \mathrm{~m}^{2}\right)$ using the Modification of Diet in Renal Disease study equation. The nearest creatinine value within 1 year prior to or after the index date was extracted. Outpatient prescriptions for oral anticoagulation (warfarin, dabigatran, apixaban, or rivaroxaban) were obtained from Mayo Clinic and Olmsted Medical Center.

The primary outcome was thromboembolic events including ischemic stroke, transient ischemic attack, or systemic emboli which were diagnosed using ICD-9 codes. A random sample of the thromboembolic events were verified by manual chart review and $100 \%$ of these were found to represent true outcomes. Mortality and the development of atrial fibrillation were secondary outcomes. Incident atrial fibrillation was defined using ICD-9 codes and deaths were identified from inpatient and outpatient medical records, Minnesota death certificates, and obituaries and notices of death in the local newspapers. The ICD-9 codes used to define variables and outcomes are listed in the supplemental material.

The baseline CHA2DS2-VASc-R scores were calculated at the index date by adding 1 point for the presence of renal impairment to the CHA2DS2-VASc score. The number of points awarded for renal impairment in calculating the CHA2DS2-VASc-R score was determined by comparing the hazard ratio (HR) of thromboembolism for renal impairment to the collective HR for various CHA2DS2-VASc scores. One point was chosen as the HRs for the various CHA2DS2-VASc scores roughly corresponded to the score value and the HR for renal impairment was 1.3. Similarly, the ATRIA stroke score assigns 1 point for renal impairment. ${ }^{11}$ CHA2DS2-VASc-R scores were grouped as follows: 0-2, 3-6, and 7-10. These groupings were chosen based on similar Log Rank HRs for the individual scores within the groups.

Outcomes stratified by CHA2DS2-VASc-R score groups were evaluated using KaplanMeier curves and the Log-Rank test. Multivariate modeling of outcomes was completed using Cox proportional hazard models. A $P$-value $<0.05$ was considered as statistically significant. All statistical analyses were performed using SAS version 9.4 software (SAS institute Inc. Cary NC). The relationship between baseline GFR and the study outcomes was also assessed. Baseline GFR data was grouped by CKD stages according to the 2012 Kidney Disease Improving Global Outcomes clinical practice guideline although CKD stage 3B and 3A were combined for simplicity.

\section{RESULTS}

The baseline cohort characteristics are shown in Table 1 . The population $(n=1,606)$ had a mean age of 69.8 years (standard deviation [SD] 12.6) and mean follow-up of 5.5 years (SD 3.4). There was a high degree of comorbidity within the population as defined by a high prevalence of CHA2DS2-VASc variables (mean CHA2DS2-VASc score 4.2). 207 patients 
(13\%) had renal impairment at baseline defined as CKD stage 3 or greater by ICD-9 codes. The total number of patients for which there was available GFR lab data within 1 year of the study start date was 1127 (70\%). Those with baseline renal impairment as defined by ICD-9 codes had greater Kaplan-Meier estimates of thromboembolism (31\%), mortality (57\%), and atrial fibrillation (43\%) at 7-years of follow-up compared to those without renal impairment $(17 \%, 26 \%$, and $28 \%$, respectively) with Logrank $P<0.0001$.

Table 2 shows the associated risk of incident outcomes for CHA2DS2-VASc scores 2 to 5 and 6 to 9 (CHA2DS2-VASc score 0-1 is the comparison), renal impairment and anticoagulation. Renal impairment as a single variable was predictive of mortality (HR 2.06, $95 \%$ confidence interval $[\mathrm{CI}] 1.64$ to $2.60, P<0.001)$. There was a trend towards predicting thromboembolism and atrial fibrillation (HR $1.34,95 \%$ CI 0.96 to $1.87, P=0.08$; and HR $1.31,95 \%$ CI 0.98 to $1.74, P=0.07)$. As we have previously described, the CHA2DS2-VASc score was predictive of the 3 outcomes, where increasing risk was seen with higher scores and this was most substantial for the outcomes of death and thromboembolism.

Mortality, thromboembolism and incident atrial fibrillation correlated significantly with increasing CHA2DS2-VASc-R score (Figure 2). The respective C-statistics mildly increased for all outcomes with the addition of renal impairment to the CHA2DS2-VASc score, although this improvement was not significant for atrial fibrillation. Specifically, the Cindices for thromboembolism, survival and atrial fibrillation increased from 0.72 to 0.73 ( $P=0.006), 0.70$ to 0.72 ( $P<0.001)$, and 0.606 to $0.607(P=0.20)$, respectively.

Table 3 shows the associated risk of study outcomes for CHA2DS2-VASc scores and CKD stages. CKD stages 4 and 5 were combined into 1 group given the relatively small percentage of patients in both groups compared to the other CKD stages. CKD stage was associated with a significantly increased risk of mortality and non-significant slightly increased risks of thromboembolism and incident atrial fibrillation. Although nonsignificant, HRs for thromboembolism were on the order of 2 for individual CKD stages compared to CKD stage 1. Figure 3 shows the relationship between various CKD stages and the outcomes. A separate analysis was completed assuming those patients without lab data had normal renal function (supplemental material). In this analysis $(n=1,606), C K D$ stage was associated with a nonsignificant nominally increased risk of mortality and thromboembolism.

\section{DISCUSSION}

Our results show that adding a point for renal impairment to the CHA2DS2-VASc score improves prediction of thromboembolism and mortality in those without atrial fibrillation in a population with implantable cardiac devices. However, the magnitude of change in Cstatistics was small. Associations between renal impairment and increased primary outcomes were significant for mortality and trending towards significance for thromboembolism and atrial fibrillation.

The risk of stroke is elevated in the setting of CKD. ${ }^{12}$ Some prior evidence supports adding renal function to thromboembolic risk prediction schemas in those with atrial fibrillation, ${ }^{8}$ 
however, this has not been evaluated in populations without atrial fibrillation. The CHA2DS2-VASc score is commonly employed to risk-stratify patients with atrial fibrillation for anticoagulation to prevent stroke. Our recent study found that the CHA2DS2-VASc score remained predictive of thromboembolic events and mortality in a population without atrial fibrillation with implantable cardiac devices. ${ }^{4}$ Studies in populations with prior stroke have also found that the CHA2DS2-VASc score was predictive of short- and long-term stroke outcomes in patients without atrial fibrillation. ${ }^{13-15}$ Increasing evidence supports a relationship between stroke and atrial inflammation, fibrosis, and filling pressure. ${ }^{16-18}$ Atrial fibrosis and inflammation may contribute to both the pathophysiology of atrial fibrillation and thromboembolism and perhaps explain the lack of temporal relation between stroke events and paroxysms of atrial fibrillation or atrial tachyarrhythmias detected by implantable cardiac devices. ${ }^{19,20}$

Our findings in a population without atrial fibrillation further question a causal relationship between atrial fibrillation and stroke. The ASSERT and IMPACT studies, which did not show that atrial tachyarrhythmias precede embolic events, also weaken the case for causality. Specifically, only $8 \%$ of patients in the ASSERT study had subclinical atrial fibrillation within the 30 days preceding thromboembolic events, ${ }^{21}$ and only $29 \%$ of thromboembolic events followed atrial tachyarrhythmias in the IMPACT study. ${ }^{22}$ Furthermore, the risk of stroke remains elevated in patients with atrial fibrillation even after sinus rhythm is maintained. ${ }^{23}$ Successful ablation of atrial fibrillation does not cease progression of atrial fibrosis. ${ }^{24}$ Fibrotic atrial cardiomyopathy may explain the aforementioned observations. ${ }^{25}$ We are increasingly recognizing the importance of atrial fibrosis as it pertains to stroke. ${ }^{18}$ The CHADS2 and CHA2DS2-VASc scores have been shown to correlate with atrial fibrosis and inflammatory markers in patients with atrial fibrillation. ${ }^{26,27}$ Perhaps with further research, the clinical focus will be less on documenting atrial fibrillation prior to initiating anticoagulation, and more on conducting a comprehensive risk assessment for stroke including markers of atrial fibrosis in addition to clinical variables. The addition of renal impairment to thromboembolism prediction tools may provide greater value in populations without atrial fibrillation when making this assessment given the absence of atrial fibrillation as a strong risk factor.

Our results support further study whether those without atrial fibrillation and particularly high CHA2DS2-VASc-R scores might benefit from some degree of anticoagulation. Others have questioned whether patients without atrial fibrillation at high risk of stroke would benefit from anticoagulation citing atrial fibrotic cardiomyopathy as a possible underlying progressive cause of stroke and a future therapeutic target. ${ }^{28}$ More liberal prescribing of anticoagulation may be seen in the future, particularly given the greater efficacy and lower risk of bleeding seen with newer oral agents. ${ }^{29}$ We feel that larger, prospective studies are needed to further evaluate our findings and the effect of anticoagulation on incident thromboembolism before recommending the CHA2DS2-VASc-R score to risk stratify patients without atrial fibrillation for anticoagulation.

Limitations of the study include that it is retrospective. The population had implantable cardiac devices and a high degree of comorbidity, which limits generalization of the results. ICD-9 diagnosis codes were used to define outcomes, atrial fibrillation, renal impairment 
and CHA2DS2-VASc components. Cardiac device data was not manually interrogated; therefore, the assessment regarding some fraction of patients as either not having or developing atrial fibrillation may be inaccurate. Specifically, atrial fibrillation in the population may be over or underrepresented depending on the device settings. The size of the population with renal impairment was relatively small $(n=207$ patients [13\%]) compared to the total population evaluated, which may account for the non-significant improvement in prediction for thromboembolism and atrial fibrillation. A minority of the population received oral anticoagulation ( $8 \%$ at baseline and $14 \%$ during the study period).

The present study shows that adding a point for renal impairment to the CHA2DS2-VASc score significantly improved prediction of thromboembolism and mortality in a population with implantable cardiac devices without atrial fibrillation, although the magnitude of improvement was small. Development of atrial fibrillation correlated with increasing CHA2DS2-VASc-R score. Our findings warrant further research to establish if some degree of anticoagulation is of benefit in patients without atrial fibrillation and high CHA2DS2VASc-R scores.

\section{Supplementary Material}

Refer to Web version on PubMed Central for supplementary material.

\section{ACKNOWLEDGEMENTS}

The authors gratefully acknowledge the assistance provided by Barbara Abbott and Gary Larson with the Rochester Epidemiology Project search planning and data acquisition.

FUNDING

This study was made possible using the resources of the Rochester Epidemiology Project, which is supported by the National Institute on Aging of the National Institutes of Health under Award Number R01 AG034676.

\section{REFERENCES}

1. Lip GY, Nieuwlaat R, Pisters R, Lane DA, Crijns HJ. Refining clinical risk stratification for predicting stroke and thromboembolism in atrial fibrillation using a novel risk factor-based approach: the euro heart survey on atrial fibrillation. Chest 2010;137:263-272. [PubMed: 19762550]

2. Friberg L, Rosenqvist M, Lip GY. Evaluation of risk stratification schemes for ischaemic stroke and bleeding in 182678 patients with atrial fibrillation: the Swedish Atrial Fibrillation cohort study. European heart journal 2012;33:1500-1510. [PubMed: 22246443]

3. Kannel WB, Abbott RD, Savage DD, McNamara PM. Epidemiologic features of chronic atrial fibrillation: the Framingham study. N Engl J Med 1982;306:1018-1022. [PubMed: 7062992]

4. Parsons C, Patel SI, Cha S, Shen WK, Desai S, Chamberlain AM, Luis SA, Aguilar MI, Demaerschalk BM, Mookadam F, Shamoun F. CHA2DS2-VASc Score: A Predictor of Thromboembolic Events and Mortality in Patients With an Implantable Monitoring Device Without Atrial Fibrillation. Mayo Clin Proc 2017;92:360-369. [PubMed: 28259228]

5. Szymanski FM, Lip GY, Filipiak KJ, Platek AE, Hrynkiewicz-Szymanska A, Opolski G. Stroke Risk Factors Beyond the CHA(2)DS(2)-VASc Score: Can We Improve Our Identification of "High Stroke Risk" Patients With Atrial Fibrillation? The American journal of cardiology 2015;116:17811788. [PubMed: 26434516]

6. Roldan V, Marin F, Manzano-Fernandez S, Fernandez H, Gallego P, Valdes M, Vicente V, Lip GY. Does chronic kidney disease improve the predictive value of the CHADS2 and CHA2DS2-VASc 
stroke stratification risk scores for atrial fibrillation? Thrombosis and haemostasis 2013;109:956960. [PubMed: 23572113]

7. Piccini JP, Stevens SR, Chang Y, Singer DE, Lokhnygina Y, Go AS, Patel MR, Mahaffey KW, Halperin JL, Breithardt G, Hankey GJ, Hacke W, Becker RC, Nessel CC, Fox KA, Califf RM, Committee RAS, Investigators. Renal dysfunction as a predictor of stroke and systemic embolism in patients with nonvalvular atrial fibrillation: validation of the R(2)CHADS(2) index in the ROCKET AF (Rivaroxaban Once-daily, oral, direct factor Xa inhibition Compared with vitamin K antagonism for prevention of stroke and Embolism Trial in Atrial Fibrillation) and ATRIA (AnTicoagulation and Risk factors In Atrial fibrillation) study cohorts. Circulation 2013;127:224-232. [PubMed: 23212720]

8. Aspberg S, Chang Y, Atterman A, Bottai M, Go AS, Singer DE. Comparison of the ATRIA, CHADS2, and CHA2D2DS2-VASc stroke risk scores in predicting ischaemic stroke in a large Swedish cohort of patients with atrial fibrillation. Eur Heart J 2016;37:3203-3210. [PubMed: 26941204]

9. St Sauver JL, Grossardt BR, Yawn BP, Melton LJ, 3rd, Pankratz JJ, Brue SM, Rocca WA. Data resource profile: the Rochester Epidemiology Project (REP) medical records-linkage system. Int J Epidemiol 2012;41:1614-1624. [PubMed: 23159830]

10. Camm AJ, Corbucci G, Padeletti L. Usefulness of continuous electrocardiographic monitoring for atrial fibrillation. The American journal of cardiology 2012;110:270-276. [PubMed: 22503584]

11. Singer DE, Chang Y, Borowsky LH, Fang MC, Pomernacki NK, Udaltsova N, Reynolds K, Go AS. A new risk scheme to predict ischemic stroke and other thromboembolism in atrial fibrillation: the ATRIA study stroke risk score. J Am Heart Assoc 2013;2:e000250. [PubMed: 23782923]

12. Dad T, Weiner DE. Stroke and Chronic Kidney Disease: Epidemiology, Pathogenesis, and Management Across Kidney Disease Stages. Semin Nephrol 2015;35:311-322. [PubMed: 26355250]

13. Ntaios G, Lip GY, Makaritsis K, Papavasileiou V, Vemmou A, Koroboki E, Savvari P, Manios E, Milionis H, Vemmos K. CHADS(2), CHA(2)S(2)DS(2)-VASc, and long-term stroke outcome in patients without atrial fibrillation. Neurology 2013;80:1009-1017. [PubMed: 23408865]

14. Tu HT, Campbell BC, Meretoja A, Churilov L, Lees KR, Donnan GA, Davis SM. Pre-stroke CHADS2 and CHA2DS2-VASc scores are useful in stratifying three-month outcomes in patients with and without atrial fibrillation. Cerebrovascular diseases 2013;36:273-280. [PubMed: 24135809]

15. Vemmos K, Ntaios G, Savvari P, Vemmou AM, Koroboki E, Manios E, Kounali A, Lip GY. Stroke aetiology and predictors of outcome in patients with heart failure and acute stroke: a 10-year follow-up study. European journal of heart failure 2012;14:211-218. [PubMed: 22200911]

16. Guo Y, Lip GY, Apostolakis S. Inflammation in atrial fibrillation. Journal of the American College of Cardiology 2012;60:2263-2270. [PubMed: 23194937]

17. Schnabel RB, Larson MG, Yamamoto JF, Kathiresan S, Rong J, Levy D, Keaney JF, Jr., Wang TJ, Vasan RS, Benjamin EJ. Relation of multiple inflammatory biomarkers to incident atrial fibrillation. The American journal of cardiology 2009;104:92-96. [PubMed: 19576326]

18. Hirsh BJ, Copeland-Halperin RS, Halperin JL. Fibrotic atrial cardiomyopathy, atrial fibrillation, and thromboembolism: mechanistic links and clinical inferences. Journal of the American College of Cardiology 2015;65:2239-2251. [PubMed: 25998669]

19. Healey JS, Connolly SJ, Gold MR, Israel CW, Van Gelder IC, Capucci A, Lau CP, Fain E, Yang S, Bailleul C, Morillo CA, Carlson M, Themeles E, Kaufman ES, Hohnloser SH, Investigators A. Subclinical atrial fibrillation and the risk of stroke. N Engl J Med 2012;366:120-129. [PubMed: 22236222]

20. Glotzer TV, Daoud EG, Wyse DG, Singer DE, Ezekowitz MD, Hilker C, Miller C, Qi D, Ziegler PD. The relationship between daily atrial tachyarrhythmia burden from implantable device diagnostics and stroke risk: the TRENDS study. Circ Arrhythm Electrophysiol 2009;2:474-480. [PubMed: 19843914]

21. Brambatti M, Connolly SJ, Gold MR, Morillo CA, Capucci A, Muto C, Lau CP, Van Gelder IC, Hohnloser SH, Carlson M, Fain E, Nakamya J, Mairesse GH, Halytska M, Deng WQ, Israel CW, Healey JS, Investigators A. Temporal relationship between subclinical atrial fibrillation and embolic events. Circulation 2014;129:2094-2099. [PubMed: 24633881] 
22. Martin DT, Bersohn MM, Waldo AL, Wathen MS, Choucair WK, Lip GY, Ip J, Holcomb R, Akar JG, Halperin JL, Investigators I. Randomized trial of atrial arrhythmia monitoring to guide anticoagulation in patients with implanted defibrillator and cardiac resynchronization devices. Eur Heart J 2015;36:1660-1668. [PubMed: 25908774]

23. Wyse DG, Waldo AL, DiMarco JP, Domanski MJ, Rosenberg Y, Schron EB, Kellen JC, Greene HL, Mickel MC, Dalquist JE, Corley SD, Atrial Fibrillation Follow-up Investigation of Rhythm Management I. A comparison of rate control and rhythm control in patients with atrial fibrillation. N Engl J Med 2002;347:1825-1833. [PubMed: 12466506]

24. Teh AW, Kistler PM, Lee G, Medi C, Heck PM, Spence SJ, Morton JB, Sanders P, Kalman JM. Long-term effects of catheter ablation for lone atrial fibrillation: progressive atrial electroanatomic substrate remodeling despite successful ablation. Heart Rhythm 2012;9:473-480. [PubMed: 22079885]

25. Kottkamp H Fibrotic atrial cardiomyopathy: a specific disease/syndrome supplying substrates for atrial fibrillation, atrial tachycardia, sinus node disease, AV node disease, and thromboembolic complications. J Cardiovasc Electrophysiol 2012;23:797-799. [PubMed: 22554187]

26. Daccarett M, Badger TJ, Akoum N, Burgon NS, Mahnkopf C, Vergara G, Kholmovski E, McGann CJ, Parker D, Brachmann J, Macleod RS, Marrouche NF. Association of left atrial fibrosis detected by delayed-enhancement magnetic resonance imaging and the risk of stroke in patients with atrial fibrillation. Journal of the American College of Cardiology 2011;57:831-838. [PubMed: 21310320]

27. Akdag S, Simsek H, Sahin M, Akyol A, Duz R, Babat N. Association of epicardial adipose tissue thickness and inflammation parameters with CHA2DS2-VASASc score in patients with nonvalvular atrial fibrillation. Ther Clin Risk Manag 2015;11:1675-1681. [PubMed: 26609234]

28. Bayes de Luna A, Baranchuk A, Martinez-Selles M, Platonov PG. Anticoagulation in patients at high risk of stroke without documented atrial fibrillation. Time for a paradigm shift? Ann Noninvasive Electrocardiol 2017;22.

29. Dogliotti A, Paolasso E, Giugliano RP. Novel oral anticoagulants in atrial fibrillation: a metaanalysis of large, randomized, controlled trials vs warfarin. Clin Cardiol 2013;36:61-67. [PubMed: 23338902] 


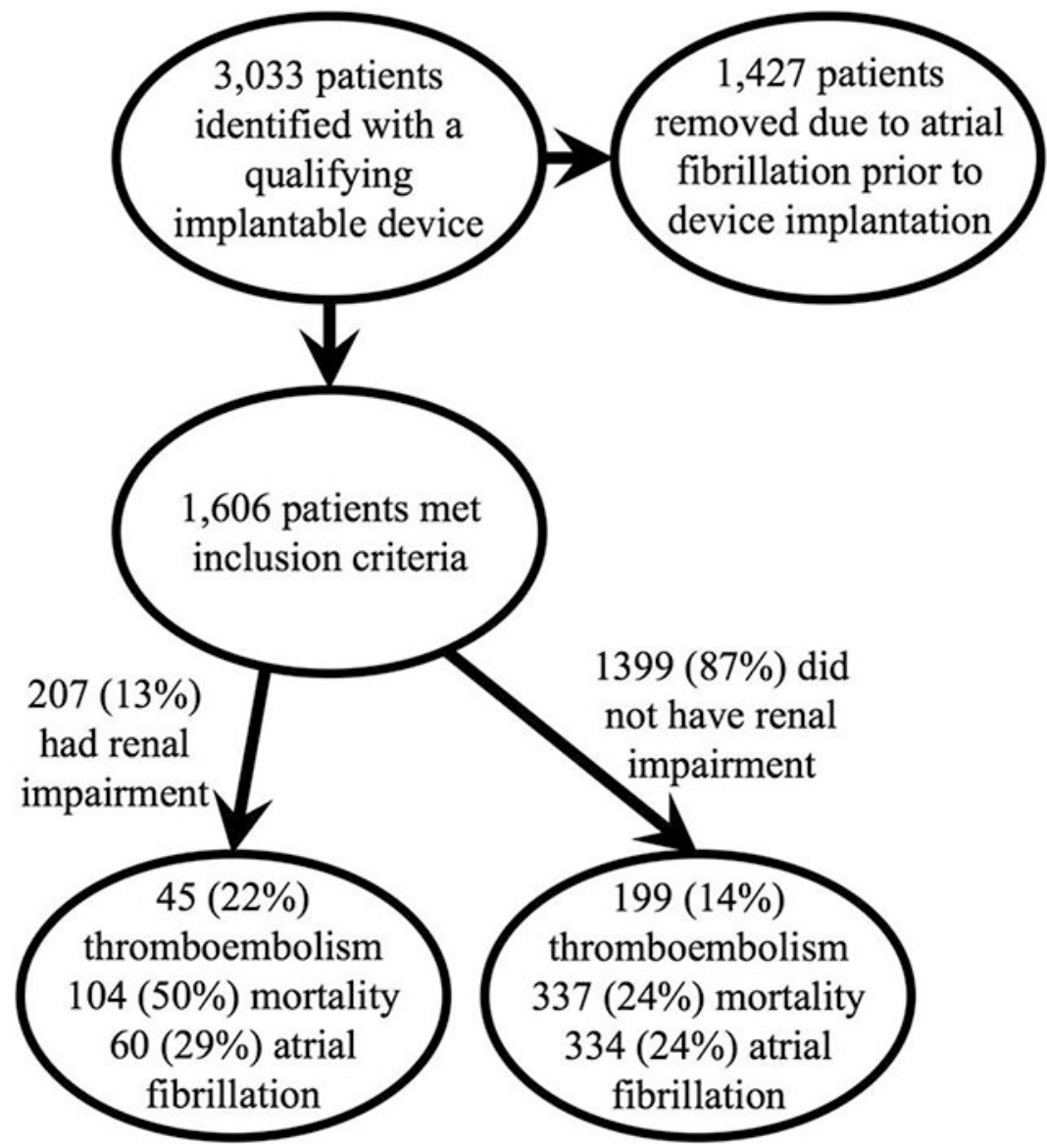

Figure 1:

Flow diagram of study sample identification and descriptive statistics for outcomes stratified by baseline renal impairment. 


$\mathrm{CHA}_{2} \mathrm{DS}_{2}-\mathrm{VASC}+\mathrm{R}: \quad 0-2 \longrightarrow 3-6 \longrightarrow$ 7-10
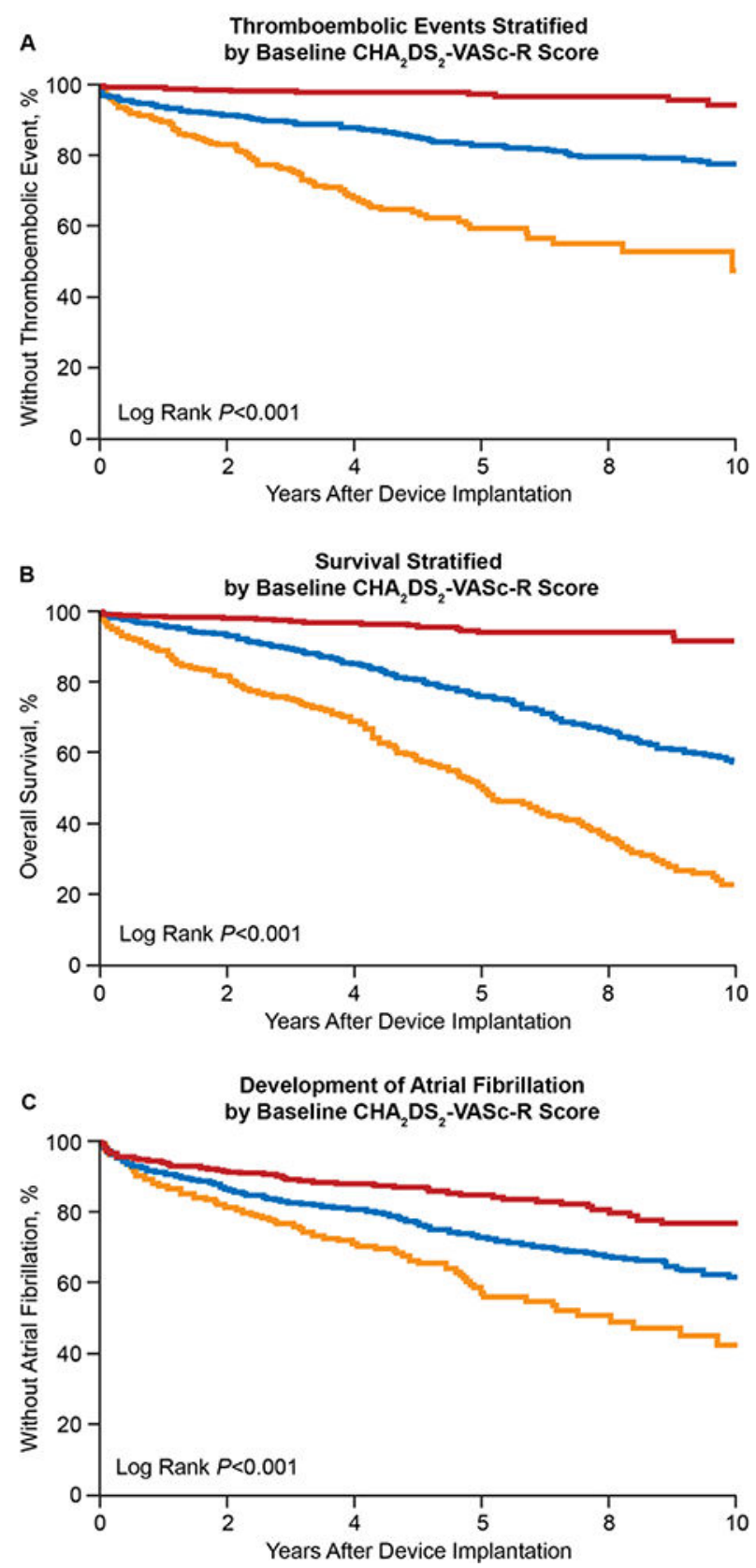

Figure 2:

Study outcomes stratified by baseline CHA2DS-VASc-R scores. 
a. Thromboembolic Events Stratified by Baseline CKD stage

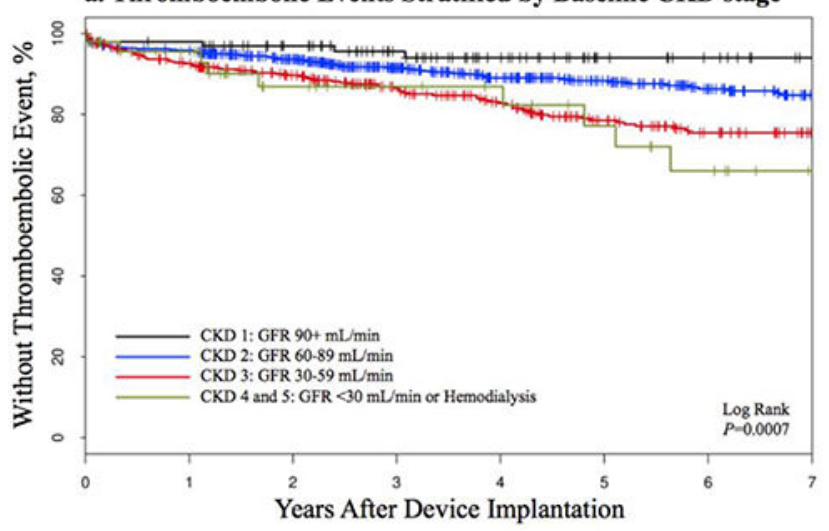

b. Survival Stratified by Baseline CKD stage

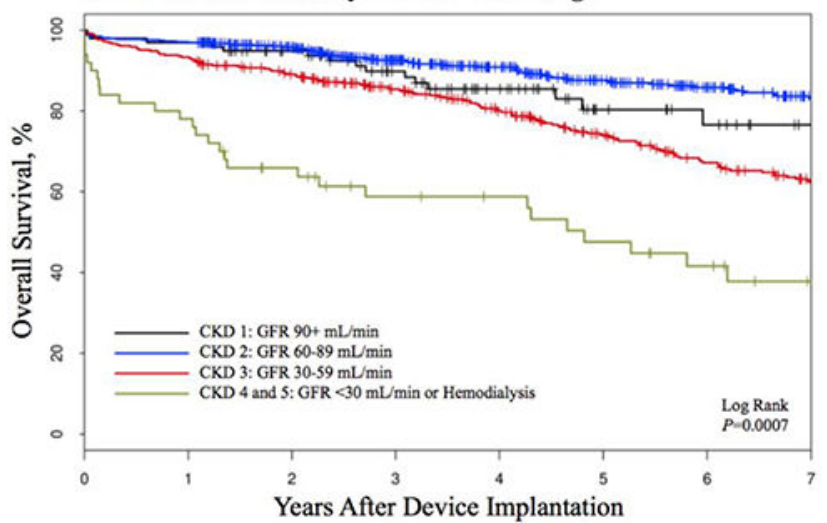

c. Incident Atrial Fibrillation Stratified by Baseline CKD stage

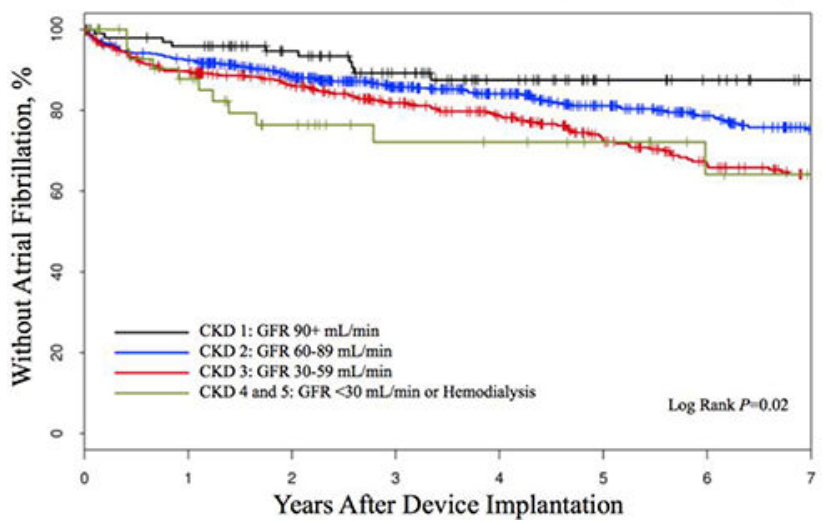

Figure 3:

Study outcomes stratified by baseline CKD stage.

$\mathrm{CKD}=$ chronic kidney disease, $\mathrm{GFR}=$ glomerular filtration rate 
Table 1.

Baseline characteristics.

\begin{tabular}{lrr}
\hline Variable & \multicolumn{2}{c}{ Overall } \\
(N=1606)
\end{tabular}

$\mathrm{SD}=$ standard deviation

*

Defined by GFR data. The total number of patients with GFR data available was 1127 (70\%)

${ }^{\dagger}$ CKD stage 3 or greater defined by ICD-9 codes for the total population $(n=1,606)$. 
Table 2.

Risk of outcomes by CHA2DS2-VASc score and renal impairment. ${ }^{*}$

\begin{tabular}{lccrr}
\hline $\begin{array}{l}\text { Outcome } \\
\text { Evaluated Variable }\end{array}$ & $\begin{array}{l}\text { Hazard } \\
\text { Ratio }\end{array}$ & $\begin{array}{c}\text { 95\% CI } \\
\text { Lower }\end{array}$ & Upper & P-value \\
\hline Death & & & & \\
CHA2DS2-VASc 2-5 & 5.16 & 2.65 & 10.06 & $<.0001$ \\
\hline CHA2DS2-VASc 6-9 & 11.26 & 5.75 & 22.05 & $<.0001$ \\
Renal Impairment & 2.06 & 1.64 & 2.60 & $<.0001$ \\
Baseline Anticoagulation & 0.76 & 0.53 & 1.09 & 0.13 \\
\hline Thromboembolic Events & & & & \\
CHA2DS2-VASc 2-5 & 3.52 & 1.64 & 7.56 & 0.001 \\
CHA2DS2-VASc 6-9 & 9.26 & 4.29 & 19.99 & $<.0001$ \\
Renal Impairment & 1.34 & 0.96 & 1.87 & 0.08 \\
Baseline Anticoagulation & 1.89 & 1.33 & 2.69 & 0.0004 \\
\hline Atrial Fibrillation & & & & \\
CHA2DS2-VASc 2-5 & 1.59 & 1.09 & 2.32 & 0.02 \\
CHA2DS2-VASc 6-9 & 2.40 & 1.61 & 3.57 & $<.0001$ \\
Renal Impairment & 1.31 & 0.98 & 1.74 & 0.07 \\
Baseline Anticoagulation & 1.29 & 0.93 & 1.78 & 0.12 \\
\hline & & & & \\
\hline
\end{tabular}

CKD stage 3 or greater defined by ICD-9 codes. 
Table 3.

Risk of outcomes by CHA2DS2-VASc score and chronic kidney disease stage. ${ }^{*}$

\begin{tabular}{|c|c|c|c|c|}
\hline \multirow{2}{*}{$\begin{array}{l}\text { Outcome } \\
\text { Evaluated Variable }\end{array}$} & \multirow{2}{*}{$\begin{array}{l}\text { Hazard } \\
\text { Ratio }\end{array}$} & \multicolumn{2}{|c|}{$95 \% \mathrm{CI}$} & \multirow[b]{2}{*}{ P-value } \\
\hline & & Lower & Upper & \\
\hline \multicolumn{5}{|l|}{ Death } \\
\hline CHA2DS2-VASc Score & 1.35 & 1.27 & 1.43 & $<.0001$ \\
\hline Chronic Kidney Disease Stage & 1.58 & 1.33 & 1.89 & $<.0001$ \\
\hline CHA2DS2-VASc 3-6 & 6.55 & 2.42 & 17.68 & 0.0002 \\
\hline CHA2DS2-VASc 7-9 & 15.08 & 5.48 & 41.46 & $<.0001$ \\
\hline Chronic Kidney Disease 2 & 0.69 & 0.40 & 1.20 & 0.19 \\
\hline Chronic Kidney Disease 3 & 1.40 & 0.82 & 2.38 & 0.22 \\
\hline Chronic Kidney Disease 4-5 & 2.74 & 1.46 & 5.14 & 0.002 \\
\hline \multicolumn{5}{|l|}{ Thromboembolic Events } \\
\hline CHA2DS2-VASc Score & 1.38 & 1.28 & 1.49 & $<.0001$ \\
\hline Chronic Kidney Disease Stage & 1.09 & 0.87 & 1.36 & 0.45 \\
\hline CHA2DS2-VASc 3-6 & 3.10 & 1.36 & 7.09 & 0.007 \\
\hline CHA2DS2-VASc 7-9 & 9.35 & 3.94 & 22.16 & $<.0001$ \\
\hline Chronic Kidney Disease 2 & 1.82 & 0.79 & 4.18 & 0.16 \\
\hline Chronic Kidney Disease 3 & 2.23 & 0.97 & 5.14 & 0.06 \\
\hline Chronic Kidney Disease 4-5 & 1.96 & 0.69 & 5.58 & 0.21 \\
\hline \multicolumn{5}{|l|}{ Atrial Fibrillation } \\
\hline CHA2DS2-VASc Score & 1.15 & 1.08 & 1.22 & $<.0001$ \\
\hline Chronic Kidney Disease Stage & 1.15 & 0.95 & 1.38 & 0.15 \\
\hline CHA2DS2-VASc 3-6 & 1.56 & 0.98 & 2.49 & 0.06 \\
\hline CHA2DS2-VASc 7-9 & 2.60 & 1.52 & 4.42 & 0.001 \\
\hline Chronic Kidney Disease 2 & 1.62 & 0.88 & 3.02 & 0.12 \\
\hline Chronic Kidney Disease 3 & 1.88 & 1.01 & 3.51 & 0.048 \\
\hline Chronic Kidney Disease 4-5 & 1.92 & 0.82 & 4.47 & 0.13 \\
\hline
\end{tabular}

* Defined by GFR data. 